\title{
The immune-inflammatory response of oligodendrocytes in a murine model of preterm white matter injury: the role of TLR3 activation
}

\author{
Marta Boccazzi $\mathbb{B}^{1,2,8}$, Juliette Van Steenwinckel ${ }^{1,2}$, Anne-Laure Schang ${ }^{3}$, Valérie Faivre ${ }^{1,2}$, Tifenn Le Charpentier ${ }^{1,2}$, \\ Cindy Bokobza ${ }^{1,2}$, Zsolt Csaba ${ }^{1,2}$, Claudia Verderio ${ }^{4}$, Marta Fumagalli5, Shyamala Mani ${ }^{1,2,6}$ and Pierre Gressens (i) ${ }^{1,2,7}$
}

\begin{abstract}
A leading cause of preterm birth is the exposure to systemic inflammation (maternal/fetal infection), which leads to neuroinflammation and white matter injury (WMI). A wide range of cytokines and chemokines are expressed and upregulated in oligodendrocytes (OLs) in response to inflammation and numerous reports show that OLs express several receptors for immune related molecules, which enable them to sense inflammation and to react. However, the role of OL immune response in WMI is unclear. Here, we focus our study on toll-like receptor-3 (TLR3) that is activated by double-strand RNA (dsRNA) and promotes neuroinflammation. Despite its importance, its expression and role in OLs remain unclear. We used an in vivo mouse model, which mimics inflammation-mediated WMI of preterm born infants consisting of intraperitoneal injection of IL-1 $\beta$ from P1 to P5. In the IL-1 $\beta$-treated animals, we observed the upregulation of T/r3, IL-1 $\beta, I F N-\beta, C C / 2$, and $C x C / 10$ in both PDGFRa+ and O4+ sorted cells. This upregulation was higher in $\mathrm{O} 4+$ immature OLs (immOLs) as compared to PDGFRa+ OL precursor cells (OPCs), suggesting a different sensitivity to neuroinflammation. These observations were confirmed in OL primary cultures: cells treated with TLR3 agonist Poly (I:C) during differentiation showed a stronger upregulation of $\mathrm{CCl} 2$ and $\mathrm{C} x \mathrm{Cl} 10$ compared to cells treated during proliferation and led to decreased expression of myelin genes. Finally, OLs were able to modulate microglia phenotype and function depending on their maturation state as assessed by qPCR using validated markers for immunomodulatory, proinflammatory, and anti-inflammatory phenotypes and by phagocytosis and morphological analysis. These results show that during inflammation the response of OLs can play an autonomous role in blocking their own differentiation: in addition, the immune activation of OLs may play an important role in shaping the response of microglia during inflammation.
\end{abstract}

\section{Introduction}

Maternal/foetal infection can perturb development increasing the risk for permanent neurological and neuropsychological disorders ${ }^{1,2}$. Sterile systemic inflammation induced by IL- $1 \beta$ injections in newborn mouse results in hypomyelination and is a model for diffuse

Correspondence: Pierre Gressens (pierre.gressens@inserm.fr)

${ }^{1}$ Université de Paris, Inserm UMR 1141 NeuroDiderot, F-75019 Paris, France

${ }^{2}$ PremUP, F-75006 Paris, France

Full list of author information is available at the end of the article

These authors contributed equally: Shyamala Mani, Pierre Gressens

Edited by A. Verkhratsky white matter injury (WMI) observed in human preterm infants $^{3}$. Myelination requires proliferation of oligodendrocyte precursor cells (OPCs) and maturation of premyelinating oligodendrocytes (OLs) into myelinating OLs, a process blocked in this model ${ }^{4}$. Although previous studies have shown a causal link between microglial activation and myelination defects ${ }^{5}$ how systemic inflammation leads to a block in OL maturation is not completely understood.

Innate immune response is the first line of defense against infection and plays an important role in sterile inflammation through the production of type I IFNs and

\section{(c) The Author(s) 2021}

(c) (i) Open Access This article is licensed under a Creative Commons Attribution 4.0 International License, which permits use, sharing, adaptation, distribution and reproduction cc) in any medium or format, as long as you give appropriate credit to the original author(s) and the source, provide a link to the Creative Commons license, and indicate if changes were made. The images or other third party material in this article are included in the article's Creative Commons license, unless indicated otherwise in a credit line to the material. If material is not included in the article's Creative Commons license and your intended use is not permitted by statutory regulation or exceeds the permitted use, you will need to obtain permission directly from the copyright holder. To view a copy of this license, visit http://creativecommons.org/licenses/by/4.0/. 
proinflammatory cytokines ${ }^{6}$. Toll-like receptor-3 (TLR3) is a member of the TLR family of innate immune response receptors and has a unique expression pattern and subcellular localization ${ }^{7,8}$. Aside from double-strand RNA (dsRNA) derived from infectious agents ${ }^{9}$, it can be activated by damaged cells caused by sterile inflammation ${ }^{10-12}$. TLR3 expression in the neonatal brain has been demonstrated $^{13}$ and its activation during development results in several cellular and behavioral abnormalities ${ }^{14-17}$. Alterations in TLR3 have been reported in WMI, including receptor upregulation in the lesion area and in the adjacent WM regions, altered localization in neurons and increased expression in reactive astrocytes ${ }^{13,18}$.

Here we studied whether TLR3 in OLs could contribute to maturation block during perinatal inflammation. We tested whether inflammation has a differential effect on different populations of immature OLs ${ }^{3}$ both in vivo and following TLR3 activation in vitro. Finally, we tested whether immune response of OLs can modulate microglial activity.

\section{Materials and methods}

In vivo IL-1 $\beta$ administration

Experimental protocols were approved by the institutional guidelines of the Institut National de la Santé et de la Recherche Scientifique (Inserm, France) and met the guidelines for the United States Public Health Service's Policy on Humane Care and Use of Laboratory Animals (NIH, Bethesda, Maryland, USA). The protocol was approved by the Bichat-Robert Debre ethical committee under the reference 2011-14/ 676-0053. OF1 strain pregnant female were purchased from Charles River (L'Arbresle, France) and male pups were randomly assigned to treatment or control group. IL-1 $\beta$ exposure was carried out as previously described ${ }^{3}$. In brief, mice received twice a day from postnatal day 1 (P1) to $\mathrm{P} 4$ and once on P5 a $5-\mu \mathrm{L}$ intraperitoneal injection of $10 \mathrm{ng} / \mathrm{g} /$ injection recombinant mouse IL- $1 \beta$ in phosphate buffered saline (PBS; R\&D Systems, Minneapolis, MN, USA) or PBS alone. Animals were sacrificed $4 \mathrm{~h}$ after the morning injection of IL-1 $\beta$ at P3, P5, and P10.

\section{PDGFRa + and $04+$ magnetic-activated cell sorting}

At P3, P5, and P10, brains were collected for cell dissociation and for PDGFR $\alpha$-positive and O4-positive cell enrichment using a magnetic coupled antibody (MACS, Miltenyi Biotec, Bergisch Gladbach, Germany), as previously described $^{4,19}$ and according to the manufacturer's protocol. In brief, pooled brains $(n=4$ at P3, $n=3$ at P5, and $n=2$ at P10) were dissociated using the Papain Neural Tissue Dissociation Kit. Brain homogenate cells were first incubated with the anti-O4 microBeads antibody and $\mathrm{O} 4+$ cells were isolated. $\mathrm{O} 4+$ cells are both PDGFR $\alpha$ - and PDGFR $\alpha+$ (but hereafter referred as
$\mathrm{O} 4+$ cells). Flow through containing the $\mathrm{O} 4-$ fraction were incubated with the anti-PDGFR $\alpha$ microBeads antibody; thus, these cells were PDGFR $\alpha+/ \mathrm{O} 4-$ (referred as PDGFR $\alpha+$; Fig. 1A).

\section{Flow cytometry}

Cell cycle was analyzed by 5-ethynyl-2-deoxyuridine (EdU) incorporation and DNA content measurement using 647 EdU Click Proliferation Kit (BD Biosciences, San Diego, CA, USA) and propidium iodide (PI, Sigma, St Louis, MO, USA). EdU was added to sorted cell during $3 \mathrm{~h}$; click reaction and PI staining were performed according to manufacturer recommendations. The gating strategy selected FSC and SSC singlets, followed by selection of cells in "G0/G1", "S", and "G2/M" phases. Cells with low DNA content were defined as apoptotic.

\section{Primary cultures}

Brains from P5 pups were dissociated with Papain Neural Tissue Dissociation Kit ${ }^{4,20}$ and OPCs or microglia were enriched by MACS using the anti-PDGFR $\alpha$ or antiCD11b MicroBeads, respectively.

\section{OPCs primary culture}

After cell sorting, OPCs were cultured as previously described $^{21,22}$. Cells were treated with the TLR3 agonist Poly(I:C) (PIC, $50 \mathrm{ug} / \mathrm{ml}$; Sigma) and kept in proliferation or differentiation medium for additional $72 \mathrm{~h}(72 \mathrm{~h})$. In selected experiments, cells were treated with IFNY (either 8 or $16 \mathrm{ng} / \mathrm{mL}$; Miltenyi).

To assess proliferation, cells were maintained in proliferation or differentiation condition for $72 \mathrm{~h}$ and incubated with $5 \mu \mathrm{M}$ EdU for the last $16 \mathrm{~h}$ at $37^{\circ} \mathrm{C}$. EdU incorporation was detected with the Click-iT EdU Alexa Fluor-594 Imaging Kit according to manufacturer's protocol.

\section{Multiplex cytokine/chemokine assay}

Proliferation and differentiation medium harvested from OL culture after $72 \mathrm{~h}$ was centrifuged briefly to remove particulates $(600 \times g$ for $10 \mathrm{~min})$. CCL2 (MCP- 1$)$, CCL3 (MIP-1a) CCL5 (RANTES), and CCL11 (Eotaxin) levels were measured using a Bio-plex 200 according to the manufacturer's instructions (Biorad laboratories, Marnes la Coquette, France). All samples were run in duplicates and data were analyzed with the Bio-Plex Manager software.

\section{Microglia primary culture}

After cell sorting, microglia were cultured as previously described $^{5}$. For medium conditioning, proliferation and differentiation medium was collected from OL culture after $24 \mathrm{~h}$ or $72 \mathrm{~h}$ and spun at $600 \times g$ for $10 \mathrm{~min}$ to remove cell debris. $0.8 \mathrm{~mL}$ (27\%) of conditioned medium $(\mathrm{CM})$ was added to $2.2 \mathrm{~mL}$ of microglia medium and $1 \mathrm{~mL}$ 


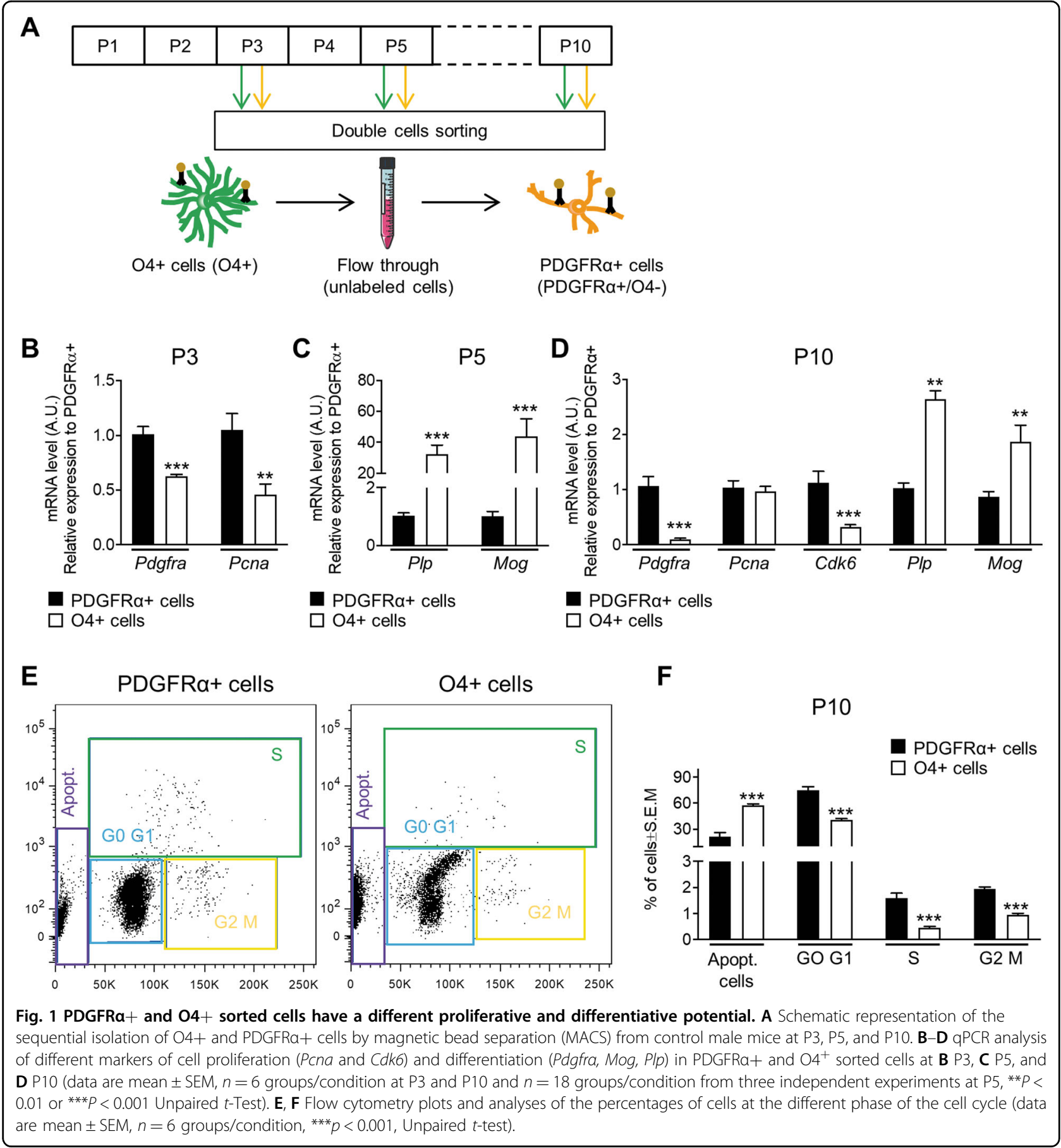

added to each well for stimulation. For PIC condition, PIC was added at the concentration present in the CM directly to microglia media $(13.3 \mu \mathrm{g} / \mathrm{mL}$ corresponding to $27 \%$ of $50 \mu \mathrm{g} / \mathrm{mL}$ ) and at two different dilutions: $4.4 \mu \mathrm{g} / \mathrm{mL}$ and $1.33 \mu \mathrm{g} / \mathrm{mL}$. Twenty hour after stimulation RNA was extracted.

To evaluate phagocytosis, microglia were plated in $\mu$ Slide 8-Well Glass Bottom chamber slides (Ibidi, BioValley; 100,000 cells/well). Fluorescent latex beads were activated by incubation with 50\% FBS for one hour at 37 degrees with frequent agitation. 100 beads/cells were added to microglia after $21 \mathrm{~h}$ of stimulation with PIC or the CM. After $3 \mathrm{~h}$ cells were washed twice with PBS and then fixed with $4 \%$ PFA.

\section{Immunocytochemistry}

Primary cells were fixed (in 4\% PFA) and immunolabelled as previously described ${ }^{23}$. Mouse anti-NG2 (1:200; 
ab50009, Abcam, France), rabbit anti-GPR17 (1:100, custom antibody produced by PRIMM, Milan, Italy), mouse anti-O4 (1:100, MAB 1326, Bio-Techne SAS, France) mouse anti-MBP (1:200, MAB382, Merck Chimie SAS, France), and goat anti-Iba1 (1:500, abcam) primary antibodies were used. Fluorescent anti-goat, anti-rabbit or anti-mouse IgG (all 1:600, $1 \mathrm{~h}$ at RT; Life Technologies, USA) secondary antibodies were used. Double labeling with anti-O4 was performed using detergent-free buffers. Nuclei were counterstained with DAPI (Sigma-Aldrich). Images were acquired using a fluorescent microscope (Nikon Eclipse Ti-E) and all the analyses were performed with the ImageJ (NIH). For oligodendrocytes, in each coverslip the total number of DAPI+ nuclei and the number of MBP + cells were determined in 20-25 optical fields chosen according to a pre-established scheme. Measurements of the total surface area and the surface area covered by MBP immunostaining were performed using an appropriate macro design.

Fluorescent microbeads were quantified on IBA1+ cells. Results were expressed as the percentage of phagocytic cells (number of cell with beads/number of total IBA1+ cells). For microglial morphological analysis between 257 and 349 single cells/group have been analyzed and on the basis of IBA1 immunostaining cells area, cells perimeter, and cells sphericity using the relation $4 \pi \times$ (area/perimeter 2$)^{24}$ have been measured.

\section{RNA extraction and qPCR}

Preparation of samples for qRT- PCR, primer design, and PCR protocol were similar to that previously described ${ }^{19,20}$. Primer sequences are given in Supplementary Table 1. The relative expression of genes of interest was determined relative to expression of the reference gene, Ribosomal Protein L13 (Rpl13). Analyses were performed with the Biorad CFX manager 2.1 software and a relative quantification approach was used, according to the 2-ddCT method ${ }^{25}$.

\section{Statistical analysis}

All data are reported as means \pm SEM. Statistical analysis of all data was performed using GraphPad PRISM version 6.0 (San Diego, CA). Data were tested for normality using the Kolmogorov-Smirnov and Bartlet test or F-test were used to evaluate that variances were equal across groups. In case of single comparisons, the Student's $t$-test was applied whereas for more than two groups a one-way analysis of variance (ANOVA) followed by Bonferroni post-hoc multiple test was used. For all analyses, statistical significance is denoted as ${ }^{*} P<0.05,{ }^{* *} P<$ 0.01 , or ${ }^{* * *} P<0.001$. For in vivo experiments there were at least six biological replicates at each timepoint whereas in vitro experiments are performed at least in triplicate to provide adequate statistical power. No power analysis was performed. No data were excluded. No blinding was done.

\section{Results}

PDGFRa+ and 04+ cell populations have different proliferation and differentiation properties

Sequential isolation of $\mathrm{O} 4+$ and PDGFR $\alpha+/ \mathrm{O} 4-$ cells by MACS at P3, P5, and P10 (Fig. 1A). At P3, PDGFR $\alpha+$ cells had a higher expression of proliferating cell nuclear antigen (Pcna mRNA) than O4+ cells (Fig. 1B). The O4+ population, already committed towards differentiation, showed lower Pcna and Pdgfra expression (Fig. 1B). At P5 the $\mathrm{O} 4+$ population exhibited higher mRNA expression for myelin genes (Fig. 1C) and at P10 the expression of Pdgfra was strongly reduced (Fig. 1D). Of note, at this stage no difference in Pcna expression was detected between the two cell populations. However, the level of Cdk6 mRNA, whose downregulation is important for differentiation ${ }^{26}$, was significantly lower in $\mathrm{O} 4+$ cells (Fig. 1D). Reduced percentage of O4+ cells in all phases of the cell cycle was confirmed by FACS analysis (Fig. 1E, F). This was associated with a higher percentage of apoptotic $\mathrm{O} 4+$ cells, described previously ${ }^{27}$.

\section{T/r3 receptor expression is regulated in oligodendrocytes} during development and induced by neuroinflammation

Regulation of Tlr3 in naïve mice during development was characterized by qRT-PCR. At P3, Tlr3 expression was increased by 1.7 fold in $\mathrm{O} 4+$ cells compared to PDGFR $\alpha+$ cells, and the trend for higher expression persisted at P5. No difference in Tlr3 mRNA levels was seen at P10 (Fig. 2A). Next, modulation of Tlr3 in OLs during perinatal inflammation was studied (Fig. 2B, C). At P3, IL-1 $\beta$ injection increased the mRNA level of Tlr3 in $\mathrm{O} 4+$ cells but not in the PDGFR $\alpha+$ cells. At P5, IL-1 $\beta$ was also capable of inducing Tlr3 expression in PDGFR $\alpha$ + cells. At P10, no further regulation of Tlr3 mRNA was seen (Fig. 2C). As shown previously ${ }^{4}, \mathrm{O} 4+$ cells isolated from IL- $1 \beta$ animals showed reduced expression of myelin genes compared to control (Supplementary Fig. 1). Taken together these results indicate that $\mathrm{O} 4+$ cells display higher levels of Tlr3 transcript compared to PDGFR $\alpha+$ cells and selectively upregulate Tlr3 expression in response to IL- $1 \beta$ early in development. However, by P5, the PDGFR $\alpha+$ cells also increased their Tlr3 expression in response to IL- $1 \beta$.

\section{In response to neuroinflammation OLs upregulate expression of innate immunity related chemokines and cytokines}

Since OLs are capable of influencing an on-going inflammatory response ${ }^{28}$, we assessed whether PDGFR $\alpha+$ and $\mathrm{O} 4+$ cells differed in their ability to upregulate factors associated with an innate immune response (Fig. 3). IL-1 $\beta$ and type I interferons have a pivotal role in innate immunity and CCL2 and CXCL10 play a key role in immune cells recruitment ${ }^{29}$. In response to $\mathrm{IL}-1 \beta$ 
A

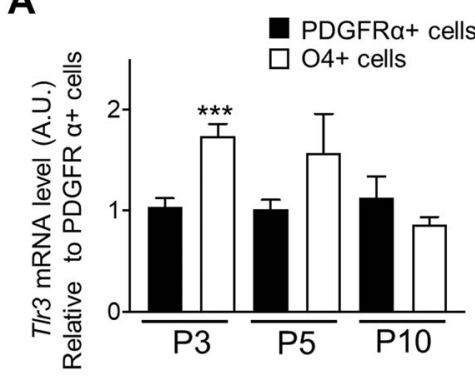

C

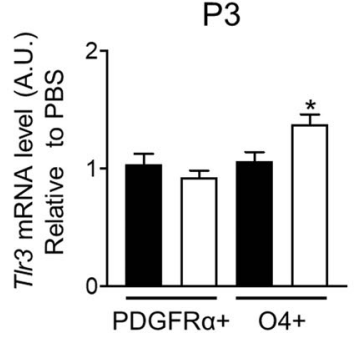

B

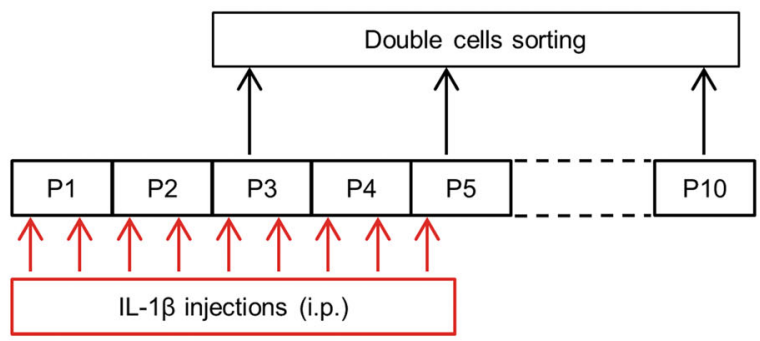

P5
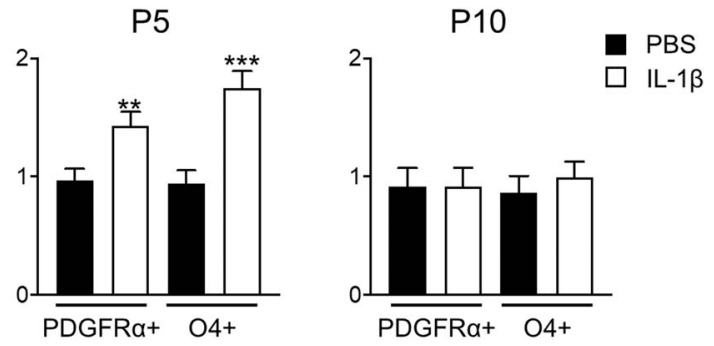

Fig. 2 TIr3 expression is differentially regulated in PDGFRa + OPCs and 04+immOLs during development and is induced after IL-1 $\beta$ treatment. A qPCR analysis of T/r3 expression in PDGFRa+ OPCs and O4+ immOLs at different postnatal days (P3, P5, and P10) form control male mice brain (data are mean \pm SEM, $n=10$ groups/condition from two independent experiments for P3 and P10 and $n=17$ groups/condition from three independent experiments at P5, ${ }^{* * *} p<0.001$ Unpaired $t$-Test). B Experimental scheme. C T/r3 expression assessed by qPCR in control and IL-1 $\beta$ treated mice in PDGFRa+ and O4+ sorted cells (data are mean \pm SEM, $n=10$ groups/condition from 2 independent experiments at P3 and P10, $n=18$ from 3 independent experiments at $P 5,{ }^{*} P<0.05,{ }^{*} P<0.01,{ }^{* *} P<0.001$ Unpaired $t$-test).

injections, PDGFR $\alpha+$ and O4+ cells upregulated $I l 1 b$ mRNA at P3 and P5 but only O4+ cells did so at P10 (Fig. 3A). Only O4+ cells responded to IL- $1 \beta$ with an increase in Ifnb1 expression at P3 and P5, and showed a tendency, although not statistically significant, to maintain elevated Ifnb1 levels at P10 (Fig. 3B). mRNA for Ccl2 and Cxcl10 (Fig. 3C, D) was increased at P3 and P5 in PDGFR $\alpha+$ and O4+ cells; however, the increase in O4+ cells was greater than that seen for the PDGFR $\alpha+$ cells. No upregulation was observed at P10. Together, these results suggest that the $\mathrm{O} 4+$ cell population has a stronger capacity to upregulate the expression of several inflammatory genes than PDGFR $\alpha+$ cells. Furthermore, in both cell populations, the immune response occurred only during the time of inflammatory challenge.

\section{TLR3 activation by Poly(l:C) during differentiation in vitro recapitulates observations in vivo}

To model the in vivo results and investigate the role of TLR3 we compared the consequences of TLR3 activation in PDGFR $\alpha+$ cells cultured either in proliferating conditions (containing bFGF and PDGF-AA) or exposed to differentiating conditions (growth factor withdrawal and addition of thyroid hormones; Fig. 4A). Poly(I:C) (PIC), a synthetic analog of dsRNA that is the natural ligand for TLR3 was used to activate the TLR3 receptor ${ }^{8}$. Cells maintained in proliferation condition expressed the OPC marker NG2 and displayed a bipolar morphology ${ }^{30}$; whereas cells shifted to differentiation started to express the immature marker GPR $17^{23,31}$ and the myelin marker MBP. Moreover, most cells in proliferation media expressed EdU with none of the cells expressing the immature marker O4; whereas, only few cells in differentiation media were EdU+ while most expressed O4 (Fig. 4B, C).

Cultured OPCs and differentiating OLs were treated with PIC and gene expression was monitored. PIC induced Tlr3 mRNA in both conditions whereas Ccl2 and Cxcl10 mRNA induction was greater in differentiated cells compared to cells kept in proliferation condition (Fig. 4D), thereby mimicking our observation in vivo. To test the specificity of this effect to TLR3, we stimulated cultures with IFN $\gamma$. Here, both undifferentiated and differentiated cells were able to upregulate $C x c l 10$ with no difference in the extent of mRNA expression (Supplementary Fig. 2). In contrast to the in vivo data, we could not reliably detect upregulation of either Illb or Ifnb1 (data not shown).

We corroborated the differential increase of $\mathrm{Ccl} 2$ mRNA in differentiating cells by showing that the amount of CCL2 protein in the medium derived from OLs maintained in differentiation condition and treated with PIC for $72 \mathrm{~h}$ was the highest. This was not the case for CCL3, CCL5, and CCL11 whose levels were either not different or higher in proliferating cells after stimulation with PIC (Fig. 5). 

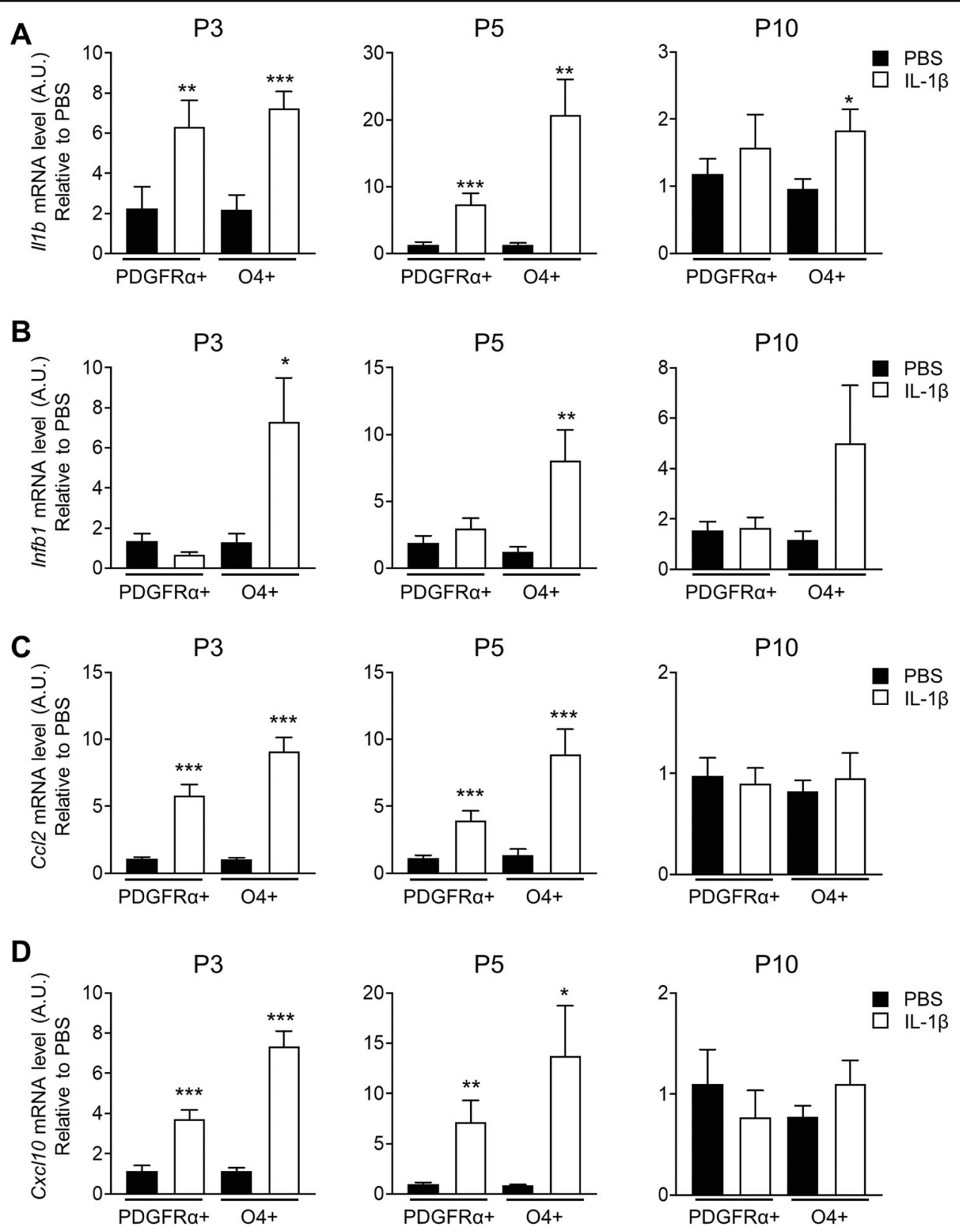

Fig. 3 Upon neuroinflammation the PDGFRa + OPCs and O4+ immOLs upregulate the expression of innate immunity related chemokines and cytokines. A-D Expression level of $1116(\mathbf{A}), 1 \mathrm{fnb} 1$ (B), CCl2 (C), and CxCl10 (D) assessed by qPCR in PDGFRa+ OPCs and O4+ immOLs MACSsorted from the brain of PBS and IL-1 $\beta$-treated mice at P3, P5, and P10 (data are mean \pm SEM, $n=12$ groups/condition from 2 independent experiments for P3 and $n=18$ groups/condition from three independent experiments for P5 and P10, ${ }^{*} P<0.05,{ }^{* *} P<0.01,{ }^{* *} P<0.001$ Unpaired $t$-test).

\section{Addition of Poly(l:C) perturbs differentiation}

mRNA encoding myelin genes $M o g$ and Plp1 were downregulated in the presence of PIC (Fig. 6A, B). Furthermore, although there was no difference in the number of MBP-positive cells (Fig. 6E), the area of MBP+ differentiated cells (Fig. 6F) and the area of MBP staining (Fig. 6G) were reduced (respectively, the yellow line and the white signal in Fig. $6 \mathrm{C}^{\prime}$ and $\mathrm{D}^{\prime}$ ), indicating that PIC stimulation limits maturation of pre-oligodendrocytes towards fully mature MBP-positive cells.

\section{Oligodendrocytes influence microglial activation in vitro}

Although it is well established that activated microglia can affect OL differentiation the reverse has not been explored much. For this, we took CM from either differentiated OLs or precursors kept in proliferating media in 


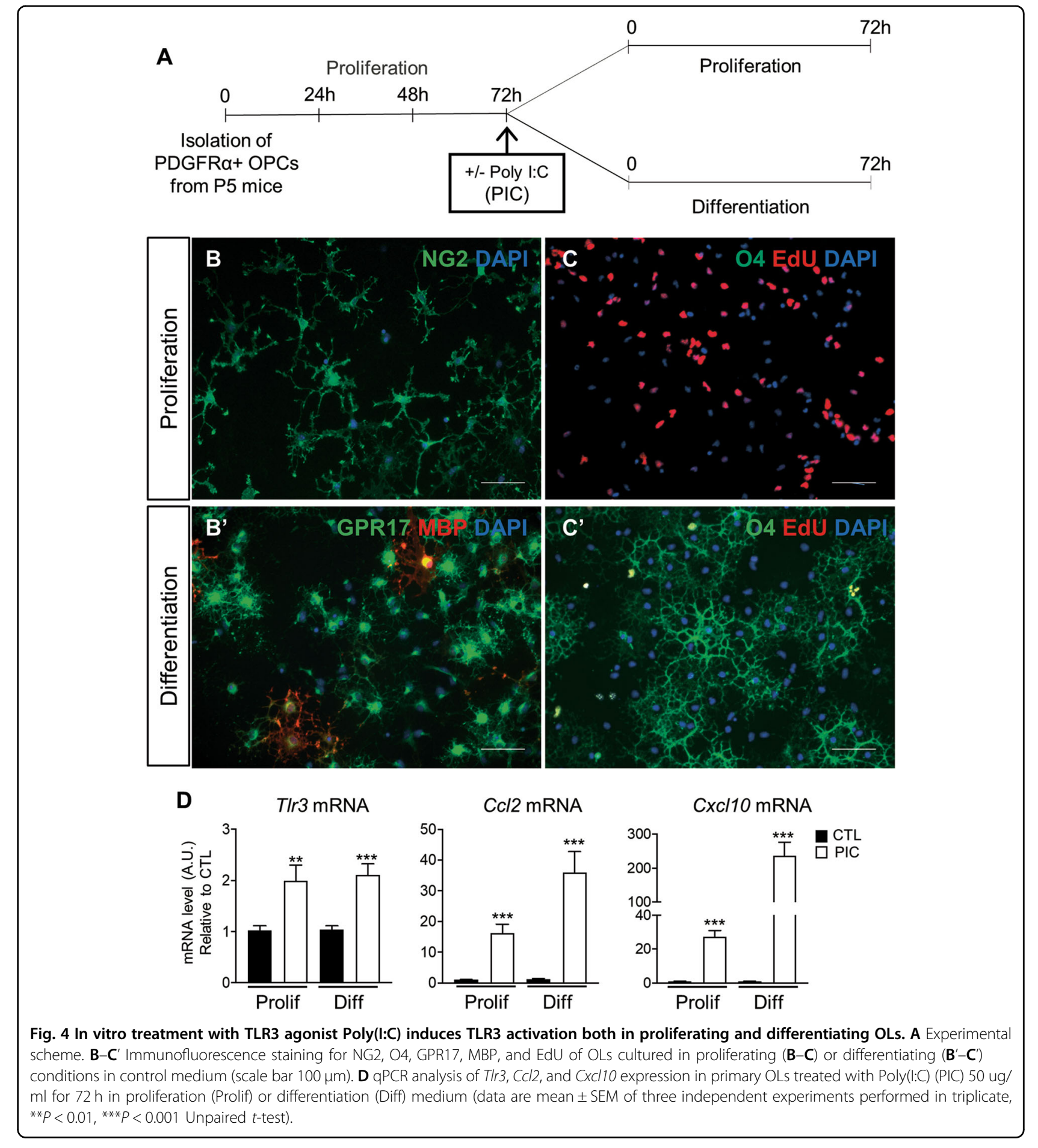

control condition or stimulated with PIC and added 27\% of $\mathrm{CM}$ to microglia media. As control, microglia was exposed to PIC $13.3 \mu \mathrm{g} / \mathrm{mL}$ (a concentration that could theoretically be present in the media not taking into account degradation of the molecule over time), $4.4 \mu \mathrm{g} /$ $\mathrm{mL}$ and $1.33 \mu \mathrm{g} / \mathrm{mL}$ (Fig. 7A).
Microglia phenotype was tested using several validated markers. Acknowledging that this is a simplification necessary to facilitate interpretation of the data, we used a nomenclature based on previous works ${ }^{5,32}$. We distinguished three phenotypes according to the mRNA expression levels of markers listed in brackets: 


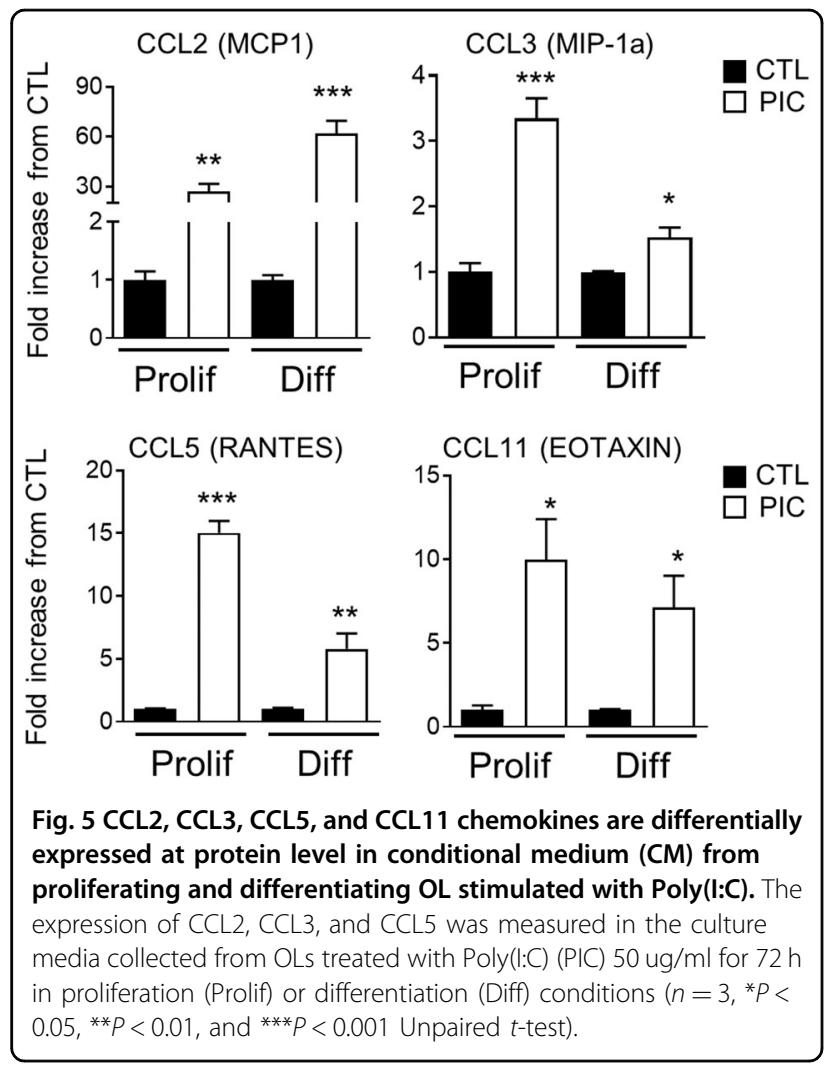

immunomodulatory (Il1rn, Socs3), proinflammatory (Nos2, Ptgs2) and anti-inflammatory (Lgals3, Igf1).

Socs3 and Il1rn mRNA levels in microglia were both specifically upregulated by CM from proliferating OPCs treated with PIC for $72 \mathrm{~h}$ compared to microglia maintained in control media (Fig. 7B-B'). Regarding Il1rn, although its mRNA level was upregulated over control also in microglia directly treated with PIC $13.3 \mu \mathrm{g} / \mathrm{mL}$, the increase was significantly higher in microglia treated with $\mathrm{CM}$ from OPCs exposed to PIC for $24 \mathrm{~h}$ and $72 \mathrm{~h}$ in proliferation medium. CM from proliferating OPCs was also significantly more efficient in inducing Il1rn expression in microglia compared to that collected from differentiating OLs both at 24 and $72 \mathrm{~h}$ (Fig. 7B').

The mRNA level of the proinflammatory marker Nos 2 in microglia was significantly upregulated only when treated with CM from differentiated cells (Fig. 7C). On the contrary, Ptgs 2 levels were induced by the two higher doses of PIC and by CM from both proliferating OPCs or differentiating OLs treated with PIC for $72 \mathrm{~h}$ (Fig. 7C').

Lgal3 was downregulated only in microglia treated with CM derived from OLs exposed to PIC for 24h (Fig. 7D); whereas $I g f 1$ reduction was seen in microglia in all the concentrations of PIC tested and with all the conditional media from OLs exposed to PIC (Fig. 7D').
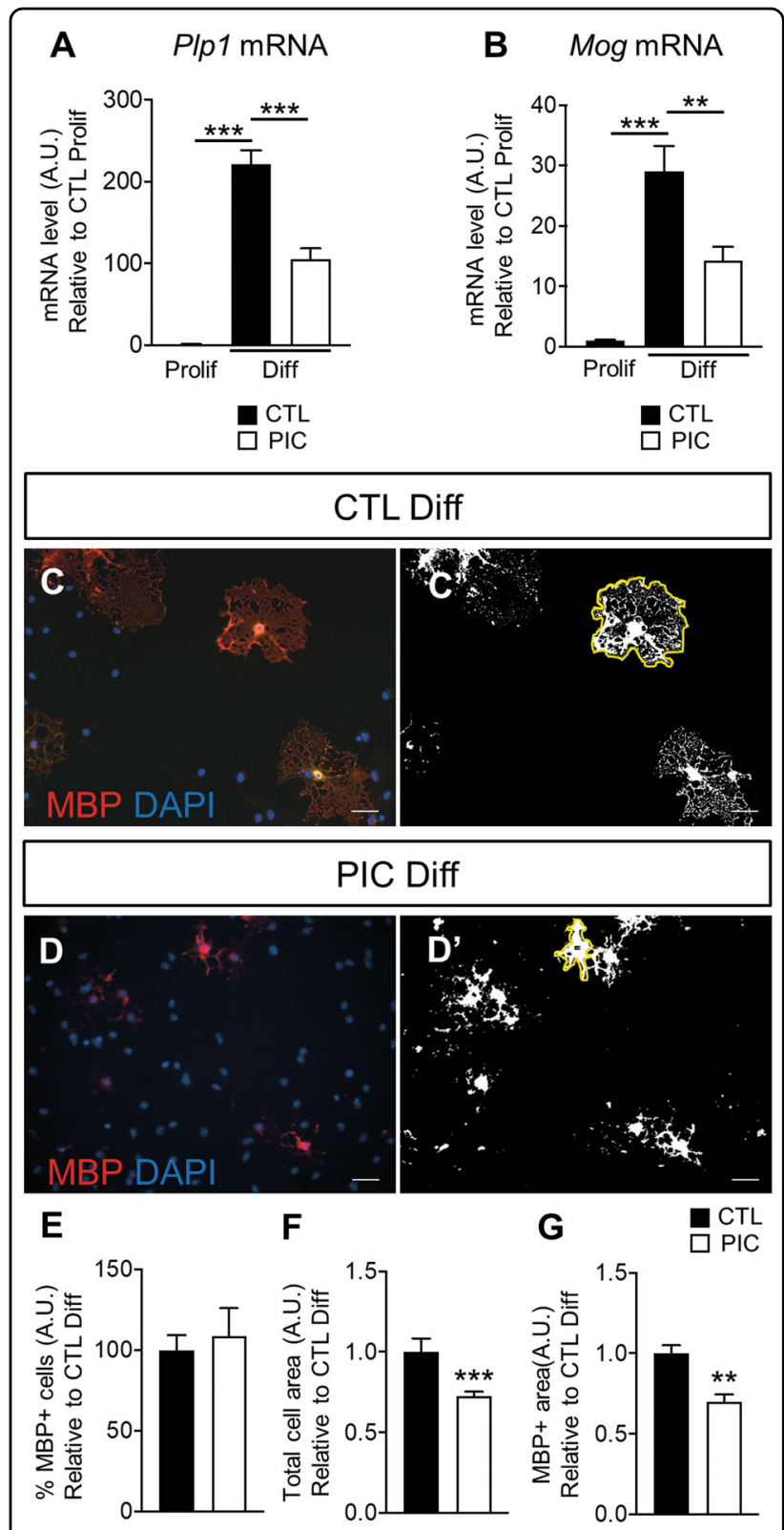

Fig. 6 Poly $(l: C)$ treatment during differentiation results in downregulation of myelin genes and defects in reaching the more mature phenotype. A-B $q P C R$ analysis of $M b p$ and $P / p 1$ in primary OLs treated with Poly(l:C) (PIC) $50 \mathrm{ug} / \mathrm{ml}$ for $72 \mathrm{~h}$ in differentiation (Diff) medium (results are the mean of four independent experiments performed in triplicate \pm SEM, ${ }^{* *} P<0.01$, ${ }^{* * *} P<0.001$ One-way Anova followed by Bonferroni's multiple comparison test). C-D' Immunofluorescent staining for MBP. To evaluate MBP morphology coloured images $(\mathbf{C}, \mathbf{D})$ have been transformed in binary with ImageJ $\left(\mathbf{C}^{\prime}, \mathbf{D}^{\prime}\right)$. E-G Histograms reported the percentage of $\mathrm{MBP}+$ cells $(\mathbf{E})$, measure of the cell area $(\mathbf{F}$, delimited with yellow line in $\mathbf{C}^{\prime}$ and $\mathbf{D}$ ) or of the area covered by MBP immunostaining (G, white signal in $\mathbf{C}^{\prime}$ and $\mathbf{D}^{\prime}$; data are mean \pm SEM, $n=9$ coverslips for each condition from three independent experiments, ${ }^{* *} P<0.01,{ }^{* *} P<0.001$ Unpaired $t$-test). Scale bar $100 \mu \mathrm{m}$ 


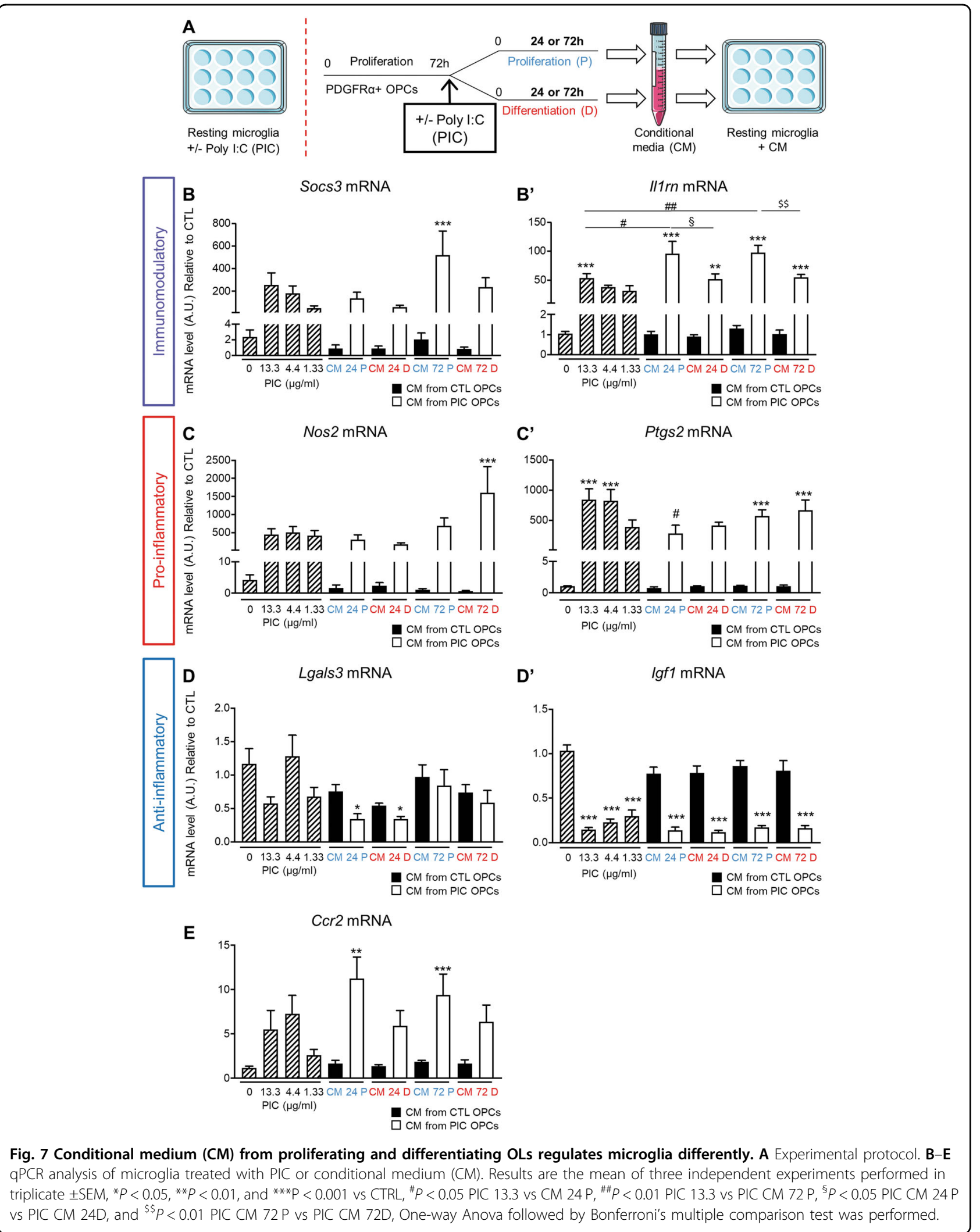




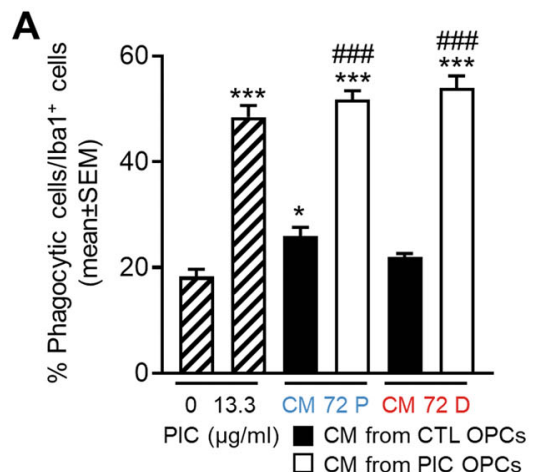

C

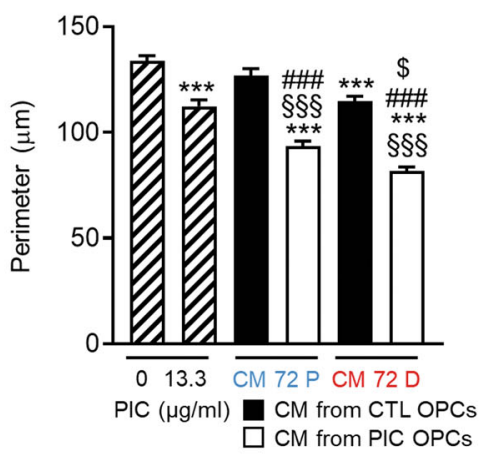

B

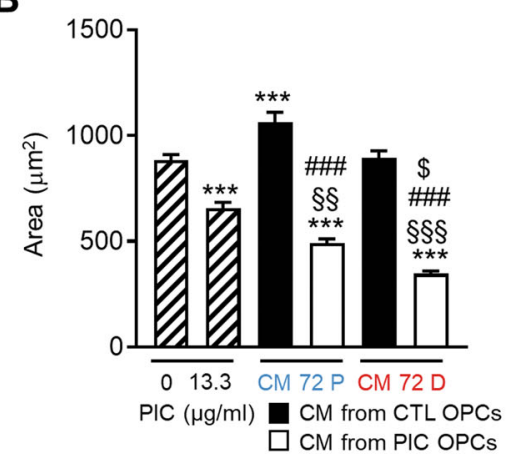

D

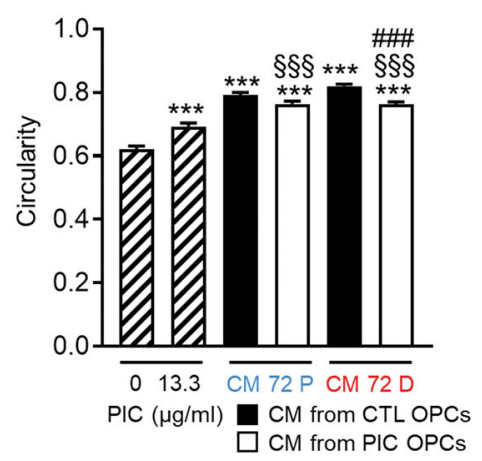

Fig. 8 Conditional medium (CM) from proliferating and differentiating OLs regulates microglia phagocytosis and cell morphology.

A Histograms reported the percentage of phagocytic cells determined as: number of cell with beads/number of total lba1+ cells. Results are the mean of three independent experiments performed in triplicate \pm SEM. B-D Microglial cell morphological changes induced by PIC or conditional medium considering cells area (B), cells perimeter $(\mathbf{C})$, and cells circularity $(\mathbf{D})$. Data are the mean of at least 250 cells/condition \pm SEM. All comparisons were performed using One-way Anova followed by Bonferroni's multiple comparison test. ${ }^{*} P<0.05$ and ${ }^{* *} P<0.001$ vs $C T R L$, ${ }^{\# \#} P<0,001$ CM from CTRL OPCs vs CM from PIC OPCs, ${ }^{\S \S} P<0.001$ and ${ }^{\S \S} P<0.01$ vs PIC, ${ }^{\$} P<0.05$ PIC CM P vs PIC CM.

Ccl2 overexpression observed herein could play a role in recruiting microglia, therefore we measured the mRNA level of $\mathrm{Ccr} 2$. We showed that $\mathrm{CM}$ from proliferating OPCs, but not differentiating OLs, was able to upregulate the expression of Ccr2 (Fig. 7E).

These data suggest that the two population of OLs differentially responded to TLR3 stimulation resulting in a different effect on microglia phenotype.

In light of the previously mentioned results, we explored whether primary microglia functions were altered by $\mathrm{CM}$ from either differentiated OLs or precursors kept in proliferating media in control condition or stimulated with PIC for $72 \mathrm{~h}$ (Fig. 8). First, phagocytosis was assessed treating the cells with specific fluorescent microbeads (Fig. 8A and Supplementary Fig. 3). We observed a significant increase in the percentage of microbeads phagocytosed per cell in microglia cultured in CM from proliferating OPCs and differentiating OLs treated with PIC both compared to cells maintained in control microglia media and to control CMs. In addition, microglia cultured in presence of PIC were also able to phagocyte microbeads. CM from unstimulated proliferating OPCs had a small effect on increasing phagocytosis capability of microglia.

Morphological analysis (Supplementary Fig. 4) showed a significant decrease in the cell body area (Fig. 8B) and perimeter (Fig. 8C) and an increase in circularity (Fig. 8D), all markers of microglia activation ${ }^{24}$, in microglia cultured in CM from both proliferating OPCs or differentiating OLs treated with PIC. Interestingly, direct challenge with PIC induced less morphology changes toward amoeboid shape compared to both CM derived from OLs treated with PIC. Finally, further confirming that the different response of the two population of OLs to TLR3 stimulation resulted in a different effect on microglia phenotype, both area and perimeter were more affected by CM from differentiating OPCs treated with PIC compared to proliferating OPCs treated with TLR3 agonist.

\section{Discussion}

Using a well-established model of perinatal neuroinflammation $^{3,5}$, we demonstrated a differential response to inflammation of PDGFR $\alpha+\mathrm{OPC}$ and $\mathrm{O} 4+\mathrm{immOL}$ populations, revealing that cells already committed 
towards a more mature phenotype have greater inflammatory capacity. Although OLs are typically considered target of detrimental inflammation, recent studies indicate they may play an active and critical role in this process ${ }^{28}$. Indeed, following cuprizone-induced demyelination, adult OPCs upregulate IL-1 $\beta$ and CCL2 becoming 'activated' and reverting their transcriptome to resemble that of neonatal state $^{33}$. Interestingly, the two proinflammatory mediators can act both in an autocrine fashion, stimulating OPCs migration, and in a paracrine way attracting microglia cells. Activation of TLR3 leading to an upregulation of IL-1 $\beta$, CCL2, and CXCL10 are also seen in models of autism and schizophrenia associated with white matter abnormalities ${ }^{2,34}$. It would be important to see whether OLs play a role in these cases.

In accordance with studies in other cell types, we showed that the TLR3 agonist PIC can transcriptionally regulate TLR3 in $\mathrm{OLs}^{35}$. Further, we showed that OL stimulation with PIC leads to differential upregulation of $\mathrm{Ccl} 2$ and Cxcl10 transcripts based on the maturation state of the cells. This differential effect was specific to TLR3 stimulation and could not be recapitulated by IFN $\gamma$. Indeed, of the several chemokines that were surveyed, CCL2 was the most abundant chemokine that was present in the PIC stimulated CM, particularly in differentiating OLs. In agreement with previous results ${ }^{36}$, PIC led to incomplete differentiation of OLs and supported the hypothesis that TLR3 activation in the differentiating population in vivo could be one pathway by which OL maturation is blocked in the IL- $1 \beta$ model, since IL-1 $\beta$ per se does not block OL differentiation ${ }^{37,38}$.

A major finding is the demonstration that the maturation state of TLR3-stimulated OLs influences OLs-microglia signalling. Socs3 and Illrn mRNA were both specifically upregulated in microglia exposed to proliferating OL-CM. In microglia SOCS3 has a critical role in inhibiting cytokine signaling and activation of microglia through suppressing $\mathrm{STAT}^{39}$. Illrn, has been shown to be neuroprotective and result in the downregulation of the proinflammatory marker $\operatorname{Nos} 2^{40}$. Here, we observed that differentiated OLs but not proliferating OPCs upregulated Nos 2 mRNA in microglia. Finally, as already observed in the cuprizone model of demyelination ${ }^{33}, C c l 2$ production by undifferentiated OLs could play a role in recruiting microglia since $\mathrm{Ccr} 2$ expression is modulated in microglia by $\mathrm{IL}-1 \beta^{19}$ and $\mathrm{CM}$ from proliferating OLs. Thus the two populations of premyelinating OLs could affect microglial differentially. Further, OL-CM stimulated with PIC increased the phagocytic activity of microglia and morphologically these cells tended to be smaller and more rounded.

In conclusion, we showed that OLs regulate important genes associated with an innate immune response during perinatal inflammation in the IL- $1 \beta$ model of WMI in preterm infants. Our results suggest a possible mechanism by which IL-1 $\beta$ could induce a maturation block in O4+ OLs.
Future studies are needed to elucidate the contribution of TLR3 and identity of the signaling pathway involved in OL maturation block in vivo.

\section{Acknowledgements}

No further Acknowledgement to declare.

\section{Funding}

This study was supported by grants from Inserm, Université de Paris, Horizon 2020 Framework Program of the European Union (grant agreement no. 874721/ PREMSTEM), ERANET-NEURON (TRAINS), ARSEP Foundation, "Brainstorming Research Assembly for Young Neuroscientists" (BraYn) Starting Grant, Università degli Studi di Milano (PSR2018 to M.F.), Merck (Grant for Multiple Sclerosis Innovation), Fondations des Gueules Cassées, Fondation Grace de Monaco, and an additional grant from "Investissement d'Avenir -ANR-11-INBS-0011-" NeurATRIS. We acknowledge financial support from the Department of Health via the National Institute for Health Research (NIHR) comprehensive Biomedical Research Centre award to Guy's \& St Thomas' NHS Foundation Trust in partnership with King's College London and King's College Hospital NHS Foundation Trust. M.B. was previously supported by a fellowship from Fondation Paralysie Cérébrale and she is currently supported by a fellowship from the Italian Multiple Sclerosis

Foundation (FISM; 2019_BS_006). The supporting bodies played no role in any aspect of study design, analysis, interpretation, or decision to publish this data.

\section{Author details \\ ${ }^{1}$ Université de Paris, Inserm UMR 1141 NeuroDiderot, F-75019 Paris, France. ${ }^{2}$ PremUP, F-75006 Paris, France. ${ }^{3}$ Université de Paris, Inserm UMR 1153, Centre de recherche en Epidémiologie et Statistiques (CRESS), Equipe HERA, Paris, France. ${ }^{4}$ CNR Institute of Neuroscience, via Vanvitelli 32, 20129 Milan, Italy. ${ }^{5}$ Department of Pharmacological and Biomolecular Sciences, Università degli Studi di Milano, via Balzaretti 9, 20133 Milan, Italy. ${ }^{6}$ Curadev Pharma Pvt. Ltd, Noida, India. ${ }^{7}$ Centre for the Developing Brain, Division of Imaging Sciences and Biomedical Engineering, King's College London, King's Health Partners, St Thomas' Hospital, London SE1 7EH, UK. ${ }^{8}$ Present address: Department of Pharmacological and Biomolecular Sciences, Università degli Studi di Milano, via Balzaretti 9, 20133 Milan, Italy}

\section{Author contributions}

M.B. and S.M. performed the animal experiments, and MACS, qRT-PCR, cell culture, and immunocytochemistry. V.F. performed the flow cytometry experiments. Z.C. provided macros for ImageJ analysis. T.L.C. performed multiplex cytokine/chemokine assay. M.B., J.V.S., A.L.S., V.F., Z.C., C.B., C.V., M.F., S.M., and P.G. participated in experimental design, interpretation of data, and preparation of the manuscript.

\section{Conflict of interest}

The authors declare that they have no conflict of interest.

\section{Ethic statement}

Experimental protocols were approved by the institutional guidelines of the Institut National de la Santé et de la Recherche Scientifique (Inserm, France) and met the guidelines for the United States Public Health Service's Policy on Humane Care and Use of Laboratory Animals ( $\mathrm{NIH}$, Bethesda, Maryland, USA). The protocol was approved by the Bichat-Robert Debre ethical committee under the reference 2011-14/676-0053.

\section{Publisher's note}

Springer Nature remains neutral with regard to jurisdictional claims in published maps and institutional affiliations.

Supplementary information The online version contains supplementary material available at https://doi.org/10.1038/s41419-021-03446-9.

Received: 2 June 2020 Revised: 13 December 2020 Accepted: 3 January 2021

Published online: 08 February 2021 


\section{References}

1. Hagberg, H., Gressens, P. \& Mallard, C. Inflammation during fetal and neonatal life: Implications for neurologic and neuropsychiatric disease in children and adults. Ann. Neurol. 71, 444-457 (2012).

2. Bokobza, C. et al. Neuroinflammation in preterm babies and autism spectrum disorders. Pediatr. Res. 85, 155-165 (2019).

3. Favrais, G. et al. Systemic inflammation disrupts the developmental program of white matter. Ann. Neurol. 70, 550-565 (2011)

4. Schang, A.-L. et al. Failure of thyroid hormone treatment to prevent inflammation-induced white matter injury in the immature brain. Brain Behav. Immun. 37, 95-102 (2014).

5. Van Steenwinckel, J. et al. Decreased microglial Wnt/ $\beta$-catenin signalling drives microglial pro-inflammatory activation in the developing brain. Brain $\mathbf{1 4 2}$ 3806-3833 (2019)

6. Ablasser, A., Hertrich, C., Waßermann, R. \& Hornung, V. Nucleic acid driven sterile inflammation. Clin. Immunol. 147, 207-215 (2013).

7. Medzhitov, R. \& Janeway, C. Innate immune recognition: mechanisms and pathways. Immunol. Rev. 173, 89-97 (2000).

8. Alexopoulou, L., Holt, A., Medzhitov, R. \& Flavell, R. Recognition of doublestranded RNA and activation of NF-kappaB by Toll. Nature 413, 732-738 (2001).

9. Matsumoto, M., Oshiumi, H. \& Seya, T. Antiviral responses induced by the TLR3 pathway. Rev. Med. Virol. 21, 67-77 (2011).

10. Tatematsu, M., Seya, T. \& Matsumoto, M. Beyond dsRNA: toll-like receptor 3 signalling in RNA-induced immune responses. Biochem. J. 458, 195-201 (2014).

11. Karikó, K., Ni, H., Capodici, J., Lamphier, M. \& Weissman, D. mRNA is an endogenous ligand for toll-like receptor 3. J. Biol. Chem. 279, 12542-12550 (2004).

12. Zhu, R. et al. Toll-like receptor 3 modulates the behavioral effects of cocaine in mice. J. Neuroinflammation 15, 93 (2018),

13. Vontell, R. et al. Toll-like receptor 3 expression in Glia and neurons alters in response to white matter injury in preterm infants. Dev. Neurosci. 35, 130-139 (2013).

14. Cameron, J. S. et al. Toll-like receptor 3 is a potent negative regulator of axonal growth in mammals. J. Neurosci. 27, 13033-13041 (2007).

15. Lathia, J. D. et al. Toll-like receptor 3 is a negative regulator of embryonic neural progenitor cell proliferation. J. Neurosci. 28, 13978-13984 (2008).

16. Meyer, U., Nyffeler, M., Yee, B. K., Knuesel, I. \& Feldon, J. Adult brain and behavioral pathological markers of prenatal immune challenge during early/ middle and late fetal development in mice. Brain Behav. Immun. 22, 469-486 (2008).

17. Ratnayake, U., Quinn, T. A., Castillo-Melendez, M., Dickinson, H. \& Walker, D. W. Behaviour and hippocampus-specific changes in spiny mouse neonates after treatment of the mother with the viral-mimetic Poly I:C at mid-pregnancy. Brain Behav. Immun. 26, 1288-1299 (2012).

18. Vontell, R. et al. Cellular mechanisms of toll-like receptor-3 activation in the thalamus are associated with white matter injury in the developing brain. J. Neuropathol. Exp. Neurol. 74, 273-285 (2015).

19. Krishnan, M. L. et al. Integrative genomics of microglia implicates DLG4 (PSD95) in the white matter development of preterm infants. Nat. Commun. 8 , 428 (2017).

20. Rangon, C. M. et al. Myelination induction by a histamine $\mathrm{H} 3$ receptor antagonist in a mouse model of preterm white matter injury. Brain Behav. Immun. 74, 265-276 (2018).
21. Bonfanti, E. et al. Abnormal upregulation of GPR17 receptor contributes to oligodendrocyte dysfunction in SOD1 G93A Mice. Int. J. Mol. Sci. 21, 2395 (2020).

22. Madsen, P. M. et al. Oligodendrocytes modulate the immune-inflammatory response in EAE via TNFR2 signaling. Brain Behav. Immun. 84, 132-146 (2020).

23. Ceruti, S. et al. Expression of the new P2Y-like receptor GPR17 during oligodendrocyte precursor cell maturation regulates sensitivity to ATP-induced death. Glia 59, 363-378 (2011).

24. Mairesse, J. et al. Oxytocin receptor agonist reduces perinatal brain damage by targeting microglia. Glia 67, 345-359 (2018).

25. Livak, K. J. \& Schmittgen, T. D. Analysis of relative gene expression data using real-time quantitative $P C R$ and the 2- $\triangle \triangle C T$ method. Methods 25, 402-408 (2001).

26. Grossel, M. J. \& Hinds, P. W. Beyond the cell cycle: a new role for cdk6 in differentiation. J. Cell. Biochem. 97, 485-493 (2006).

27. Caprariello, A. V. et al. Apoptosis of oligodendrocytes during early development delays myelination and impairs subsequent responses to demyelination. J. Neurosci. 35, 14031-14041 (2015).

28. Zeis, T., Enz, L. \& Schaeren-Wiemers, N. The immunomodulatory oligodendrocyte. Brain Res. 1641, 139-148 (2016).

29. Babcock, A. A., Kuziel, W. A., Rivest, S. \& Owens, T. Chemokine expression by glial cells directs leukocytes to sites of axonal injury in the CNS. J. Neurosci. 23, 7922-7930 (2003)

30. De Castro, F. \& Bribián, A. The molecular orchestra of the migration of oligodendrocyte precursors during development. Brain Res. Rev. 49, 227-241 (2005).

31. Fumagalli, M. et al. Phenotypic changes, signaling pathway, and functional correlates of GPR17-expressing neural precursor cells during oligodendrocyte differentiation. J. Biol. Chem. 286, 10593-10604 (2011).

32. Chhor, V. et al. Characterization of phenotype markers and neuronotoxic potential of polarised primary microglia In vitro. Brain Behav. Immun. 32, 70-85 (2013).

33. Moyon, S. et al. Demyelination causes adult CNS progenitors to revert to an immature state and express immune cues that support their migration. J. Neurosci. 35, 4-20 (2015).

34. Arrode-Brusés, G. \& Brusés, J. L. Maternal immune activation by poly(l:C) induces expression of cytokines IL-1 $\beta$ and IL-13, chemokine MCP-1 and colony stimulating factor VEGF in fetal mouse brain. J. Neuroinflammation 9, 83 (2012).

35. Fragale, A. et al. Critical role of IRF-8 in negative regulation of TLR3 expression by Src homology 2 domain-containing protein tyrosine phosphatase-2 activity in human myeloid dendritic cells. J. Immunol. 186, 1951-1962 (2011).

36. Bsibsi, M., Nomden, A., van Noort, J. M. \& Baron, W. Toll-like receptors 2 and 3 agonists differentially affect oligodendrocyte survival, differentiation, and myelin membrane formation. J. Neurosci. Res. 90, 388-398 (2012).

37. Mason, J. L., Suzuki, K., Chaplin, D. D. \& Matsushima, G. K. Interleukin-1ß promotes repair of the CNS. J. Neurosci. 21, 7046-7052 (2001).

38. Vela, J. M., Molina-Holgado, E., Arévalo-Martín, Á., Almazán, G. \& Guaza, C. Interleukin-1 regulates proliferation and differentiation of oligodendrocyte progenitor cells. Mol. Cell. Neurosci. 20, 489-502 (2002).

39. Qin, $\mathrm{H}$. et al. Signal transducer and activator of transcription-3/suppressor of cytokine signaling-3 (STAT3/SOCS3) axis in myeloid cells regulates neuroinflammation. Proc. Natl Acad. Sci. USA 109, 5004-5009 (2012).

40. Jones, N. C. et al. Antagonism of the interleukin-1 receptor following traumatic brain injury in the mouse reduces the number of nitric oxide synthase-2positive cells and improves anatomical and functional outcomes. Eur. I. Neurosci. 22, 72-78 (2005). 